\title{
Role of Neoadjuvant Chemotherapy in the Management of Advanced Ovarian Cancer
}

\author{
Dan Zhao' ${ }^{1}$, Ling-Ying Wu ${ }^{1 *}$, Xiao-Bing Wang ${ }^{2}$, Xiao-Guang Li $^{1}$
}

\begin{abstract}
Objective: To analyze efficacy of neoadjuvant chemotherapy for advanced ovarian cancer. Materials and Methods: A total of 107 patients with advanced ovarian cancer undergoing cytoreductive surgery were divided into a neoadjuvant chemotherapy group $(n=61)$ and a primary debulking group $(n=46)$ and retrospectively analyzed. Platinum-based adjuvant chemotherapy was applied to both groups after cytoreductive surgery ande overall and progression-free survival times were calculated. Results: No significant difference was observed in duration of hospitalization $(20.8 \pm 6.1 \mathrm{vs} .20 .2 \pm 5.4 \mathrm{days}, p>0.05)$. The operation time of neoadjuvant chemotherapy group was shorter than the initial surgery group $(3.1 \pm 0.7 v s .3 .4 \pm 0.8 \mathrm{~h}, p<0.05)$. There were no significant differences in median overall survival time between neoadjuvant chemotherapy group and surgery group (42 vs. 55 months, $p>0.05$ ). Similarly, there was no difference in median progression-free survival between neoadjuvant chemotherapy group and surgery group $(16 \mathrm{vs} .17 \mathrm{months}, p>0.05)$. The surgical residual tumor size demonstrated no significant difference between initial surgery and neoadjuvant chemotherapy groups $(p>0.05)$. Multivariate analysis showed that more than 3 cycles of regimen with neoadjuvant chemotherapy was associated with more resistance to chemotherapy compared with patients without receiving neoadjuvant chemotherapy (OR: 5.962, 95\% CI: 1.184-30.030, $p<0.05)$. Conclusions:Neoadjuvant chemotherapy can shorten the operation time. However, it does not improve survival rates of advanced ovarian cancer patients.
\end{abstract}

Keywords: Neoadjuvant chemotherapy - ovarian cancer - cytoreductive surgery - chemotherapy sensitivity

Asian Pac J Cancer Prev, 16 (6), 2369-2373

\section{Introduction}

Ovarian cancer is a common form of cancer in women and the main cause of death among all gynecologic cancers. Due to the lack of effective means of early detection, patients with obvious symptoms have been in advanced stage of the disease. Primary cytoreductive surgery followed by chemotherapy represents the current standard treatment for patients with advanced ovarian cancer (McGuire et al., 1996; Monk et al., 2013). Primary cytoreductive surgery aims at removing as much primary and metastatic tumor as possible in order to facilitate response to subsequent chemotherapy and improve survival (Gadducci et al., 2013; Bian et al., 2014). For patients with poor general condition or not satisfied with tumor resection, several cycles of chemotherapy before surgery was used.

Neoadjuvant chemotherapy represents any cytotoxic therapy given prior to any cytoreductive surgery (Lawton et al., 1989; Schwartz et al., 1994; Schwartz et al., 1999). Frei (1982) in 1982 originally introduced the definition of neoadjuvant chemotherapy to describe chemotherapy treatment of primary solid tumors before surgical ablation. Neoadjuvant chemotherapy followed by interval debulking surgery has been proposed as an alternative approach for the initial management of ovarian cancer, aiming at the improvement of surgical efficiency and patients' quality of life. In a retrospective study by Mazzeo et al. (2003), patients with primarily unresectable ovarian cancer received a median of four platinum-based neoadjuvant chemotherapy courses, followed by surgery and adjuvant chemotherapy in patients with an objective response or stable disease after induction of chemotherapy. In addition to improving the patient's general condition and control the pleural effusion,the main purpose of neoadjuvant chemotherapy is to improve the cytoreductive surgery satisfaction rate. Vergote et al. (2010) Of the 670 patients randomly assigned to a study treatment, The largest residual tumor was $1 \mathrm{~cm}$ or less in diameter in $41.6 \%$ of patients after primary debulking and in $80.6 \%$ of patients after interval debulking. Complete resection of all macroscopic disease (at primary or interval surgery) was the strongest independent variable in predicting overall survival.

The aim of this study was to determine the impact of the neoadjuvant chemotherapy on optimization of cytoreductive surgery, and analyze the impact of neoadjuvant chemotherapy on postoperative chemotherapy 
Dan Zhao et al

sensitivity and patient survival so as to evaluate the role of neoadjuvant chemotherapy in the management of advanced ovarian cancer compared to conventional therapy.

\section{Materials and Methods}

\section{Patients}

The study included 107 patients with advanced ovarian cancer (FIGO stages III - IV) treated at the department of Gynecologic Oncology, Tumor hospital, Chinese Academy of Medical Sciences during 2004-2010 (Table 1). 61 cases of the patients were treated with 1-5 cycles of neoadjuvant chemotherapy, 46 cases received cytoreductive surgery. For the neoadjuvant chemotherapy regimen among these 61 cases, 38 cases were treated with TC scheme, 8 cases treated with $\mathrm{CP}$ regimen, 5 cases treated with CAP regimen, 10 cases treated with TP regimen. 9 patient's received 1 course of neoadjuvant chemotherapy treatment, 41 cases with 2 courses, 11 cases with more than three courses. All the studied patients underwent combined treatment of primary cytoreductive surgery followed by adjuvant chemotherapy (treated with TC, TP, CP or CAP regimen). Optimal cytoreduction was considered to be achieved when the largest residual tumor diameter was $\leq 1 \mathrm{~cm}$. Follow up of patients were observed for more than 6 months after adjuvant chemotherapy, the median follow-up time was 48 months.

\section{Chemotherapy sensitivity}

The clinical criteria of chemosensitivity: Imaging methods and the concentraion of serum CA125 was assessed to evaluate the clinical efficacy. Clinical platinum-sensitive group had no recurrence within 6 months after the end of chemotherapy; clinical platinumresistant group had recurrence within 6 months in the progression or chemotherapy. Progression or completion of chemotherapy in cancer chemotherapy within 6 months after the recurrence. Progression in cancer chemotherapy or tumor recurrence within 6 months after chemotherapy.

\section{Statistical analysis}

All data in the study were evaluated with SPSS version 13.0 software (SPSS Inc.). The $\chi^{2}$ test was performed to determine the differences of the postoperative residual tumor cases. The t-test analysis was used to analyze the differences of the surgical bleeding, operative time and hospital stay. Overall and progression-free survival rates were assessed by the Kaplan-Meier method and Logistic regression analysis of the significance of prognostic factors (Grading, staging, pathology, surgical approach, residual tumor size and the effect of neoadjuvant chemotherapy on clinical chemosensitivity). Differences were considered significant at value of $p \leq 0.05$.

\section{Results}

The comparison of operation time, blood loss, hospital stay and the residual tumor

To evaluate the effect of neoadjuvant chemotherapy on cytoreductive operation, we compared the optimal cytoreductive operation (the largest residual tumor diameter was $\leq 1 \mathrm{~cm}$ ) rates between patients who received

Table 1. The General Information of the Patients

\begin{tabular}{|c|c|c|c|}
\hline Clinical parameters & $\begin{array}{l}\text { primary debulking } \\
\qquad(\mathrm{n}=46)\end{array}$ & $\begin{array}{l}\text { neoadjuvant } \\
\quad(\mathrm{n}=61)\end{array}$ & $p$ \\
\hline Age(years) & $57.4 \pm 9.4$ & $56.0 \pm 10.1$ & 0.465 \\
\hline Stage $(\%)$ & & & 0.079 \\
\hline IIIA & $1(2.17 \%)$ & 0 & \\
\hline IIIB & $4(8.69 \%)$ & $1(1.64 \%)$ & \\
\hline IIIC & $37(80.43 \%)$ & $47(77.05 \%)$ & \\
\hline IV & $4(8.69 \%)$ & $13(21.31 \%)$ & \\
\hline Classification & & & 0.114 \\
\hline Poorly differentiated & 32 & 48 & \\
\hline Moderately differentiated & 11 & 13 & \\
\hline Well differentiated & 3 & 0 & \\
\hline Pathological type & & & 0.465 \\
\hline Serous adenocarcinoma & 29 & 33 & \\
\hline Endometrioid adenocarcinoma & 5 & 25 & \\
\hline Clear cell carcinoma & 4 & 3 & \\
\hline Transitional cell carcinoma & 4 & 0 & \\
\hline Mixed epithelial carcinoma & 2 & 0 & \\
\hline Mucinous adenocarcinoma & 2 & 0 & \\
\hline Mucinous adenocarcinoma & 2 & 0 & \\
\hline Surgical approach & & & 0.356 \\
\hline $\begin{array}{l}\text { Total abdominal hysterectomy +bilateral salpingo-oophorectomy } \\
\text { +mentectomy+appendectomy + cytoreductive surgery }\end{array}$ & 31 & 33 & \\
\hline $\begin{array}{l}\text { Total abdominal hysterectomy +bilateral salpingo-oophorectomy } \\
\text { +mentectomy+appendectomy + cytoreductive surgery } \\
\text { + excisional biopsy of lymph node enlargement }\end{array}$ & 4 & 6 & \\
\hline $\begin{array}{l}\text { Total abdominal hysterectomy + bilateral salpingo-oophorectomy } \\
\text { +mentectomy+appendectomy + cytoreductive surgery } \\
\text { + pelvic and paraaortic lymphadenectomy }\end{array}$ & 11 & 22 & \\
\hline
\end{tabular}


neoadjuvant chemotherapy and those patients who did not receive it. In neoadjuvant chemotherapy group, optimal cytoreduction was subsequently achieved in 37 of 61 patients $(60.66 \%)$, whereas achieved in 21 of 46 patients $(45.65 \%)$ in no received neoadjuvant chemotherapy group (Table 2), with no stastistical difference. However, the cytoreductive operation rates in neoadjuvant chemotherapy group was higher than the initial surgery group. The amount of bleeding and duration of hospitalization had no significant difference betweeninitial surgery and neoadjuvant chemotherapy group $(431.5 \pm 259.3 \mathrm{ml} v s$. $382.0 \pm 231.1 \mathrm{ml}, p>0.05 ; 20.8 \pm 6.1$ days $v s .20 .2 \pm 5.4$ days, $p>0.05)$. Neoadjuvant chemotherapy group operative time was stastistically shorter than the initial surgery group $(3.1 \pm 0.7 \mathrm{~h}$ vs. $3.4 \pm 0.8 \mathrm{~h}, p<0.05)$.

\section{Comparison of the sensitivity of chemotherapy}

Logistic regression multivariate analysis was used and found the residual tumor size was correlated with clinical chemosensitivity (Table 3). Patients with tumors larger

\section{Table 2. The Comparison of Operation Time, Blood Loss, Hospital Stay and the Residual Tumor}

\begin{tabular}{lccc}
\hline Clinical parameters & $\begin{array}{c}\text { primary debulking } \\
(\mathrm{n}=46)\end{array}$ & $\begin{array}{c}\text { neoadjuvant } \\
(\mathrm{n}=61)\end{array}$ & $p$ \\
\hline Residual tumor size & & & 0.297 \\
$\leq 1 \mathrm{~cm}$ & $21(45.65 \%)$ & $37(60.66 \%)$ & \\
$1-2 \mathrm{~cm}$ & 8 & 7 & \\
$>2 \mathrm{~cm}$ & 17 & 17 & 0.3 \\
Blood loss(ml) & $431.5 \pm 259.3$ & $382.0 \pm 231.1$ & 0.041 \\
operation time (hours) & $3.4 \pm 0.8$ & $3.1 \pm 0.7$ & 0.561 \\
hospital stay (days) & $20.8 \pm 6.1$ & $20.2 \pm 5.4$ & \\
\hline
\end{tabular}

Table 3. Multivariate Logistic Regression Analysis Ofclinically Relevant Factors Affecting the Chemosensitivity

\begin{tabular}{|c|c|c|c|c|}
\hline Risk factors & wald value & OR & $95 \% \mathrm{CI}$ & $\mathrm{P}$ value \\
\hline Classification(poor vs moderate/well) & 0.018 & 1.071 & $0.393-2.920$ & 0.893 \\
\hline Staging(stage 3 vs stage 4$)$ & 2.655 & 2.86 & $0.808-10.127$ & 0.103 \\
\hline Pathology & 2.855 & & & 0.24 \\
\hline Endometrioid adenocarcinoma $v s$ serous adenocarcinoma & 0.539 & 0.674 & $0.235-1.933$ & 0.463 \\
\hline Others $* v s$ serous adenocarcinoma & 1.876 & 2.489 & $0.675-9.181$ & 0.171 \\
\hline Surgical approach & 3.745 & & & 0.154 \\
\hline 2 vs 1 & 0.33 & 0.62 & $0.121-3.169$ & 0.565 \\
\hline 3 vs 1 & 2.812 & 2.413 & $0.862-6.755$ & 0.094 \\
\hline Residual tumor size & 7.507 & & & 0.023 \\
\hline $1-2 \mathrm{~cm} v s \leq 1 \mathrm{~cm}$ & 0.001 & 0.982 & $0.246-3.915$ & 0.979 \\
\hline$>2 \mathrm{~cm} v s \leq 1 \mathrm{~cm}$ & 6.59 & 3.913 & $1.381-11.088$ & 0.01 \\
\hline Neoadjuvant chemotherapy & 4.702 & & & 0.095 \\
\hline $1-2$ courses vs 0 & 1.035 & 1.688 & $0.616-4.631$ & 0.309 \\
\hline$\geq 3$ courses $v s 0$ & 4.685 & 5.962 & $1.184-30.030$ & 0.03 \\
\hline
\end{tabular}

* Includes ovarian clear cell carcinoma, transitional cell carcinoma, mixed epithelial carcinoma and mucinous adenocarcinoma

1: Total abdominal hysterectomy +bilateral salpingo-oophorectomy+mentectomy+appendectomy + cytoreductive surgery, 2: Total abdominal hysterectomy + bilateral salpingo-oophorectomy+mentectomy+appendectomy + cytoreductive surgery + excisional biopsy of lymph node enlargement, 3: Total abdominal hysterectomy + bilateral salpingo-oophorectomy+mentectomy+appendectomy + cytoreductive surgery + pelvic and paraaortic lymphadenectomy

A

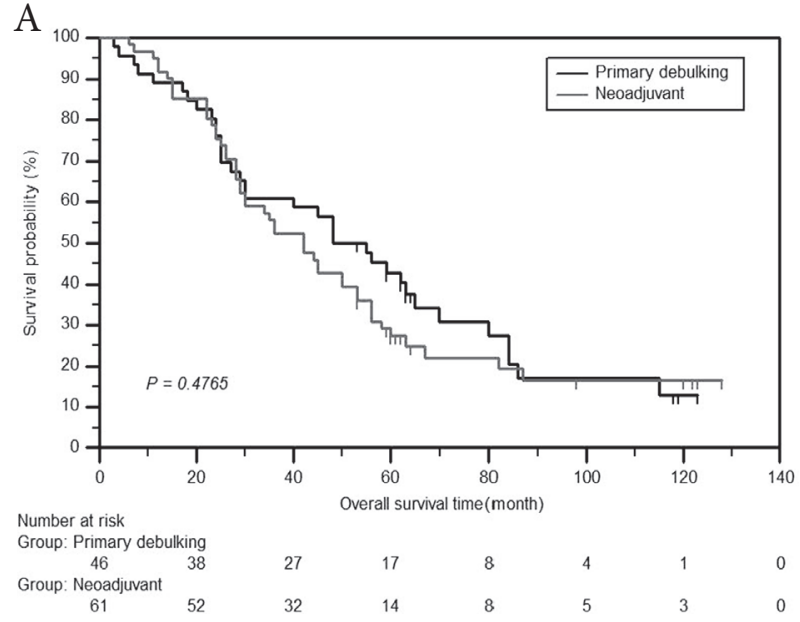

B

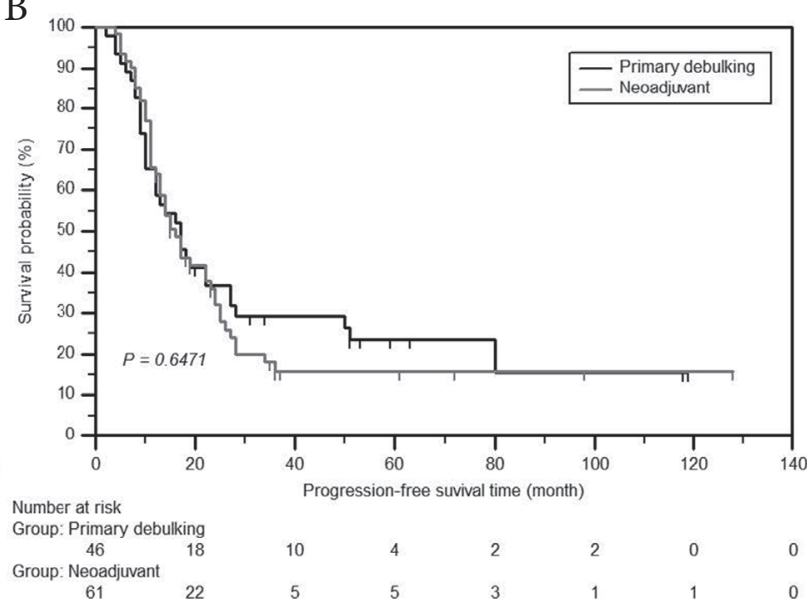

Figure 1. Overall Survival Time and Progression-Free Survival Time for Patients with Ovarian Cancer in the Primary Debulking and Neoadjuvant Chemotherapy Groups. No statistically significant difference was observed in the overall survival between the group treated with neoadjuvant chemotherapy and the no neoadjuvant chemotherapy treated group $(p=0.4765)$ (A). There was also no statistical difference was founded in the progression-free survival between these two groups $(p=0.6471)(\mathrm{B})$. 
than $2 \mathrm{~cm}$ are more prone to resistance to chemotherapy than residual tumor smaller than $1 \mathrm{~cm}$ instance (OR: 3.913 95\%CI: $1.381-11.088 p=0.010$ ).

Multivariate analysis showed that more than 3 cycles of regimen with neoadjuvant chemotherapy was prone to resistance to chemotherapy compared with patients without receiving neoadjuvant chemotherapy (OR: 5.962, 95\%CI: $1.184-30.030, p<0.05)$. However, there are no stastistical between the patients with 1-2 courses of neoadjuvant chemotherapy compared to patients without receiving neoadjuvant chemotherapy.

\section{Survival analysis}

In the neoadjuvant chemotherapy group of patients, the median overall survival was 42 months, and the median progression-free survival 16 months. In the no neoadjuvant chemotherapy group, the median overall survival was 55 months, and the median progression-free survival was 17 months. No statistically significant difference was observed in the overall survival between the group treated with neoadjuvant chemotherapy and the no neoadjuvant chemotherapy treated group $(p=0.4765)$ (Figure 1A). There was also no statistical difference was founded in the progression-free survival between these two groups $(p=0.6471)$ (Figure1B).

\section{Discussion}

Neoadjuvant chemotherapy represents a few cycles of chemotherapy given prior to tumor cytoreductive surgery which especially suitable for patients with advanced ovarian cancer. Ovarian cancer is chemotherapy sensitive epithelial tumor, total efficiency can achieve 70\% 80\% and $40 \% \sim 50 \%$ can achieve clinical complete remission after application of platinum based chemotherapy (Dewdney et al., 2010). McClug et al. (2002) confirmed the chemotherapy affection of advanced ovarian cancer through the pathological changes.

Neoadjuvant chemotherapy has the following advantages: i) neoadjuvant chemotherapy drug administration through thoracic and abdominal cavity can control the ascites, improve the general condition so as to improve the operation tolerance; ii) Destroy the liver, lung metastasis, reduce tumor staging, increase the operation feasibility; iii) Reduce tumor volume, loose tumor and normal tissue adhesions, reduce operation risk; reduction of abdominal tumor metastasis, shorten operation time, reduce intraoperative bleeding, effectively improve the cytoreductive surgery success rate; $i v$ ) obtain the operation resection specimens to evaluated the chemotherapy sensitivity; $v$ ) The neoadjuvant chemotherapy is especially suitable for advanced cancer patients with generally poor quality, large metastasis tumor, high serum CA125 level or tumor difficult to clean (Le et al., 2007; Akita et al., 2009).

Researchers showed that the neoadjuvant chemotherapy is helpful to improve the prognosis and the quality of life of patients (Sternberg et al., 1995; Chan et al., 2003; Tatematsu et al., 2013). Here, we compared the 61 cases patients received new adjuvant chemotherapy and 46 cases of patients without preoperative chemotherapy, found no significant differences in patients age, tumor stage, grade, histological type and surgical approach. Patients in the chemotherapy group, after neoadjuvant chemotherapy, the general condition was improved, created conditions for the operation, optimal cytoreductive surgery rate was $60.66 \%$, which was higher than the no received preoperative chemotherapy group, optimal cytoreductive surgery rate was $45.65 \%$, there was no significant difference between these two groups. Neoadjuvant chemotherapy can shorten the operation time, reduces operation bleeding. Barry (Rosen et al., 2014) retrospectively analyzed 326 patients with advanced ovarian cancer and the satisfaction rate of the patients received neoadjuvant chemotherapy was significantly higher than the direct surgery group $(50.1 \%$ $v s .41 .5 \% p=0.03)$. However the 7-year survival rate was significantly lower than the direct surgery group $(8.6 \% \mathrm{vs}$. $41 \% p<0.0001)$. This study compares the satisfaction of cytoreductive rate with neoadjuvant chemotherapy group of tumor size less than $1 \mathrm{~cm}$ was higher than direct surgical group, but did not reach statistical significance.Thus, the sample size in the study should be expanded.

Effect of neoadjuvant chemotherapy on survival: generally, neoadjuvant chemotherapy can reduce the pleural effusion and ascites volume, reduce tumor volume, so that to improve optimal cytoreduction opportunities of patients with advanced ovarian cancer. But studies found that neoadjuvant chemotherapy cannot improve the prognosis of patients with ovarian cancer. Inciura et al. (2006) retrospective analysis of 213 cases ovarian cancer patients received neoadjuvant chemotherapy and 361 patients treated by standard mode therapy, results showed that there was not significant differences between the two groups in OS and PFS $(p>0.05)$. Loizzi et al. (2005) founded that the median survival time, median progression free survival time and 3-years survival rate of neoadjuvant chemotherapy patients compared with the standard treatment for patients had no significant difference.

Meta-analysis (Bristow and Chi, 2006) indicated the cycle numbers of preoperative chemotherapy was negatively correlated with survival rate in ovarian cancer patients; patients with each additional 1 cycle of chemotherapy, the OS will be reduced 4.1 months. In this study, there was no statistical difference between the overall survival time and no time to disease progression, neoadjuvant chemotherapy can not improve the prognosis, consistent with previous findings.

Effect of neoadjuvant chemotherapy on drug sensitivity: Logistic regression analysis was used to analyze the multiple clinical indicators on the impact of chemotherapysensitivity. In multivariate analysis, we found patients received more than three courses of neoadjuvant chemotherapy treatmentare prone to resistance to chemotherapy (OR: $5.962,95 \% \mathrm{CI}$ : $1.184-$ $30.030, p<0.05)$. The clinical chemosensitivity had no stastistical difference of patients with 1-2 courses of neoadjuvant chemotherapy compared with patients without receiving neoadjuvant chemotherapy. Increase the courses of neoadjuvant chemotherapy medications can increase the incidence of clinical drug resistance, which may be associated with tumor acquired drug resistance, 
which may be combined with no obvious CA125 decline. The mechanism of drug resistance is influence by many factors, therefore a larger randomized clinical study need to further investigate.

\section{Acknowledgements}

The authors have no funding or financial disclosures.

\section{References}

Akita H, Doki Y, Yano M, et al (2009). Effects of neoadjuvant chemotherapy on primary tumor and lymph node metastasis in esophageal squamous cell carcinoma: additive association with prognosis. Dis Esophagus, 22, 291-7.

Bian J, Li B, Kou XJ, et al (2014). Clinical applicability of multitumor marker protein chips for diagnosing ovarian cancer. Asian Pac J Cancer Prev, 15, 8409-11.

Bristow RE, Chi DS (2006). Platinum-based neoadjuvant chemotherapy and interval surgical cytoreduction for advanced ovarian cancer: a meta-analysis. Gynecol Oncol, 103, 1070-6.

Chan YM, Ng TY, Ngan HY, et al (2003). Quality of life in women treated with neoadjuvant chemotherapy for advanced ovarian cancer: a prospective longitudinal study. Gynecol Oncol, 88, 9-16.

Dewdney SB, Rimel BJ, Reinhart AJ, et al (2010). The role of neoadjuvant chemotherapy in the management of patients with advanced stage ovarian cancer: survey results from members of the society of gynecologic oncologists. Gynecol Oncol, 119, 18-21.

Frei E, $3^{\text {rd }}(1982)$. The National Cancer Chemotherapy Program. Science, 217, 600-6.

Gadducci A, Tana R, Landoni F, et al (2013). Analysis of failures and clinical outcome of advanced epithelial ovarian cancer in patients with microscopic residual disease at second-look reassessment following primary cytoreductive surgery and first-line platinum-based chemotherapy. Eur J Gynaecol Oncol, 34, 213-7.

Inciura A, Simavicius A, Juozaityte E, et al (2006). Comparison of adjuvant and neoadjuvant chemotherapy in the management of advanced ovarian cancer: a retrospective study of 574 patients. BMC Cancer, 6, 153.

Lawton FG, Redman CW, Luesley DM, et al (1989). Neoadjuvant (cytoreductive) chemotherapy combined with intervention debulking surgery in advanced, unresected epithelial ovarian cancer. Obstet Gynecol, 73, 61-5.

Le T, Hopkins L, Faught W, et al (2007). The lack of significance of Ca125 response in epithelial ovarian cancer patients treated with neoadjuvant chemotherapy and delayed primary surgical debulking. Gynecol Oncol, 105, 712-5.

Loizzi V, Cormio G, Resta L, et al (2005). Neoadjuvant chemotherapy in advanced ovarian cancer: a case-control study. Int J Gynecol Cancer, 15, 217-23.

Mazzeo F, Berliere M, Kerger J, et al (2003). Neoadjuvant chemotherapy followed by surgery and adjuvant chemotherapy in patients with primarily unresectable, advanced-stage ovarian cancer. Gynecol Oncol, 90, 163-9.

McCluggage WG, Lyness RW, Atkinson RJ, et al (2002). Morphological effects of chemotherapy on ovarian carcinoma. J Clin Pathol, 55, 27-31.

McGuire WP, Hoskins WJ, Brady MF, et al (1996). Cyclophosphamide and cisplatin compared with paclitaxel and cisplatin in patients with stage III and stage IV ovarian cancer. $N$ Engl J Med, 334, 1-6.

Monk BJ, Dalton H, Farley JH, et al (2013). Antiangiogenic agents as a maintenance strategy for advanced epithelial ovarian cancer. Crit Rev Oncol Hematol, 86, 161-75.

Rosen B, Laframboise S, Ferguson S, et al (2014). The impacts of neoadjuvant chemotherapy and of debulking surgery on survival from advanced ovarian cancer. Gynecol Oncol, 134, $462-7$.

Schwartz PE, Chambers JT, Makuch R (1994). Neoadjuvant chemotherapy for advanced ovarian cancer. Gynecol Oncol, 53, 33-7.

Schwartz PE, Rutherford TJ, Chambers JT, et al (1999). Neoadjuvant chemotherapy for advanced ovarian cancer: long-term survival. Gynecol Oncol, 72, 93-9.

Sternberg CN, Raghaven D, Ohi Y, et al (1995). Neoadjuvant and adjuvant chemotherapy in advanced disease--what are the effects on survival and prognosis? Int J Urol, 2, 76-88.

Tatematsu N, Ezoe Y, Tanaka E, et al (2013). Impact of neoadjuvant chemotherapy on physical fitness, physical activity, and health-related quality of life of patients with resectable esophageal cancer. Am J Clin Oncol, 36, 53-6.

Vergote I, Trope CG, Amant F, et al (2010). Neoadjuvant chemotherapy or primary surgery in stage IIIC or IV ovarian cancer. N Engl J Med, 363, 943-53. 